\title{
SMALL EIGENVALUES OF THE LAPLACE OPERATOR ON COMPACT RIEMANN SURFACES ${ }^{1}$
}

\author{
BY BURTON RANDOL
}

Communicated by I. M. Singer, February 13, 1974

Let $\mathscr{S}$ be a compact Riemann surface, which we will assume to have curvature normalized to be -1 , and let $0=\lambda_{0}<\lambda_{1} \leqq \lambda_{2} \leqq \cdots$ be the eigenvalues corresponding to the problem $\Delta F+\lambda F=0$ on $\mathscr{S}$, where $\Delta$ is the Laplacian for $\mathscr{S}$. In an otherwise very interesting and useful paper [2], McKean has stated that it is always the case that $\lambda_{1} \geqq \frac{1}{4}$. In this paper, we will show that this need not be true, and that in fact, it is possible to have as many $\lambda_{n}$ 's in $\left(0, \frac{1}{4}\right)$ as one wishes. The existence of such $\lambda_{n}$ 's is of considerable interest, since they figure explicitly in the finer points of the theory of the distribution of the lengths of shortest closed geodesics in free homotopy classes on $\mathscr{S}$ (cf. [1]), as well as in the Riemann hypothesis for the Selberg zeta function corresponding to the trivial character on the fundamental group $\Gamma$ of $\mathscr{S}$.

Our point of departure will be the version of the Selberg trace formula [3] appropriate to the case at hand, which runs as follows:

Suppose $\mathscr{S}$ is the quotient of the upper half-plane by the discontinuous group $\Gamma$, consisting of, a part from the identity, hyperbolic transformations. Let $\chi$ be a character of $\Gamma$, and let $0 \leqq \lambda_{0}(\chi) \leqq \lambda_{1}(\chi) \leqq \cdots$ be the sequence of eigenvalues corresponding to the problem $\Delta F+\lambda F=0$ on $\mathscr{S}$, where the eigenfunction $F(x)$ is required to transform under $\Gamma$ by $F(\gamma x)=\chi(\gamma) F(x)$. The discussion in [3] assures that the $\lambda_{n}(\chi)$ 's are real and $\geqq 0$, and that the set of such eigenfunctions is complete in the space consisting of those measurable functions on the upper half-plane which transform in this manner, and which are $L^{2}$ over a fundamental domain of $\Gamma$. Now suppose $h(z)$ is an even function, holomorphic in a strip of the form $|\operatorname{Im} z|<$ $\frac{1}{2}+\varepsilon(\varepsilon>0)$, and satisfying a growth condition of the form $|h(z)|=$ $O\left(1+|z|^{2}\right)^{-1-\varepsilon}$, uniformly in the strip. Associate with the sequence $\lambda_{0}(\chi), \lambda_{1}(\chi), \cdots$ of eigenvalues, a sequence $R$, consisting of those numbers $r(\chi)$ that satisfy the equations $\lambda_{n}(\chi)=\frac{1}{4}+r^{2}(\chi)(n=0,1,2, \cdots)$. Apart

AMS (MOS) subject classifications (1970). Primary 30A46.

1 Professor Selberg has pointed out to me that he has previously obtained this and similar results by, among other things, a technique that directly establishes the continuous dependence of the spectrum on the character. 
from the possibility $r(\chi)=0$, the elements of $R$ will then occur in pairs, of which each member is the negative of the other, and it is always the case that each $r(\chi)$ is either real or pure imaginary, with imaginary part $\leqq \frac{1}{2}$. If one of the $\lambda_{n}(\chi)$ 's happens to be $\frac{1}{4}$, the corresponding $r(\chi)=0$ will be counted with double multiplicity in its occurrence on the left side of the trace formula.

Now all the elements of $\Gamma$ except the identity are hyperbolic. I.e., each $\gamma \in \Gamma$ is conjugate in $\operatorname{PSL}(2, R)$ to a unique transformation of the form $z \rightarrow e^{l_{\gamma}}$, where $l_{\gamma}$ is real and positive. For geometric reasons, we will call the number $l_{\gamma}$ the length of the transformation $\gamma$ (cf. [2]). Clearly $l_{\gamma}$ is the same within a conjugacy class. We will denote by $\{\gamma\}$ the conjugacy class corresponding to $\gamma$ within $\Gamma$ itself. Also, we will call $\gamma \in \Gamma$ primitive, if it is not a positive integral power of any other element of $\Gamma$. Clearly we can speak of a conjugacy class in $\Gamma$ as being primitive. The trace formula then reads

$$
\sum_{R} h(r(\chi))=\frac{A}{2 \pi} \int_{-\infty}^{\infty} h(r) r \tanh \pi r d r+\sum_{\{\}_{p}} \sum_{n=1}^{\infty} \chi^{n}(\gamma)\left(l_{\gamma} \operatorname{csch} \frac{n l_{\gamma}}{2}\right) \hat{h}\left(n l_{\gamma}\right),
$$

where $\hat{h}(x)=(2 \pi)^{-1} \int_{-\infty}^{\infty} h(r) e^{i r x} d r, A=$ the area of $\mathscr{S}$, and the outer sum is taken over all primitive conjugacy classes in $\Gamma$. Moreover, all series in the formula converge absolutely.

In what follows, we will be dealing with the transform pair

$$
h(r)=(\pi / \varepsilon)^{1 / 2} \exp \left(-r^{2} / 4 \varepsilon\right), \quad \hat{h}(x)=\exp \left(-\varepsilon x^{2}\right) \quad(\varepsilon>0)
$$

in the trace formula. For the moment, we take $\varepsilon=1$, to obtain

$$
\begin{aligned}
\pi^{1 / 2} \sum_{R} \exp \left(-r^{2}(\chi) / 4\right)= & A(4 \pi)^{-1 / 2} \int_{-\infty}^{\infty} \exp \left(-r^{2} / 4\right) r \tanh \pi r d r \\
& +\sum_{\{\gamma\}_{p}} \sum_{n=1}^{\infty} \chi^{n}(\gamma)\left(l_{\gamma} \operatorname{csch} \frac{n l_{\gamma}}{2}\right) \exp \left(-\left(n l_{\gamma}\right)^{2}\right)
\end{aligned}
$$

Observe now that the $r(\chi)$ 's all lie in the set

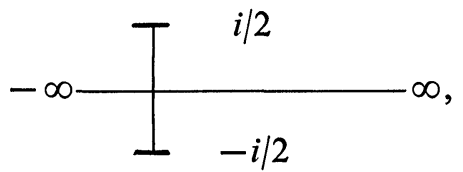

which we will break into three parts:

$I_{1}=$ the closed segment from $-i / 2$ to $i / 2$, minus the origin.

$I_{2}=$ the closed segment of the real axis from -1 to 1 .

$I_{3}=$ the union of $(-\infty,-1)$ and $(1, \infty)$. 
Then we easily infer from (1), by first taking $\chi \equiv 1$, and then observing that any other choice of $\chi$ reduces the absolute value of the double sum on the right side of (1), that

1. There exists a number $M_{1}>0$, such that the number of $r(\chi)$ 's in $I_{2}$ is $\leqq M_{1}$ for all $\chi$.

2. There exists a number $M_{2}>0$, such that $\pi^{1 / 2} \sum_{I_{3}} \exp \left(-r^{2}(\chi) / 4\right) \leqq M_{2}$ for all $\chi$.

From these facts, we conclude that

$1^{\prime} .(\pi / \varepsilon)^{1 / 2} \sum_{I_{2}} \exp \left(-r^{2}(\chi) / 4 \varepsilon\right) \leqq M_{1}(\pi / \varepsilon)^{1 / 2}$ for all $\chi$ and all $\varepsilon>0$.

$2^{\prime} .(\pi / \varepsilon)^{1 / 2} \sum_{I_{3}} \exp \left(-r^{2}(\chi) / 4 \varepsilon\right)$ can be made as small as one wishes, uniformly in $\chi$, by taking $\varepsilon$ sufficiently small.

We also observe at this point that

$$
A(4 \pi \varepsilon)^{-1 / 2} \int_{-\infty}^{\infty} \exp \left(-r^{2} / 4 \varepsilon\right) r \tanh \pi r d r \rightarrow 0 \quad \text { as } \varepsilon \rightarrow 0 .
$$

Next introducing the parameter $\varepsilon$ into the trace formula, we observe that if $\chi \equiv 1$, then

$$
\left(\frac{\pi}{\varepsilon}\right)^{1 / 2} \sum_{R} \exp \left(-r^{2}(\chi) / 4 \varepsilon\right) \sim 2\left(\frac{\pi}{\varepsilon}\right)^{1 / 2} e^{1 / 16 \varepsilon} \text { as } \varepsilon \rightarrow 0,
$$

since both $-i / 2$ and $i / 2$ then occur among the $r(\chi)$ 's.

Now choose $\varepsilon$ so small that $2(\pi / \varepsilon)^{1 / 2} e^{1 / 16 \varepsilon}$ is very large in comparison with $M_{1}(\pi / \varepsilon)^{1 / 2},(\pi / \varepsilon)^{1 / 2} \sum_{I_{3}} \exp \left(-r^{2}(\chi) / 4 \varepsilon\right)$ for any $\chi$, and

$$
A(4 \pi \varepsilon)^{-1 / 2} \int_{-\infty}^{\infty} \exp \left(-r^{2} / 4 \varepsilon\right) r \tanh \pi r d r .
$$

With $\varepsilon$ so fixed, we conclude, still assuming $\chi \equiv 1$, that

$$
\sum_{\{\gamma\}_{p}} \sum_{n=1}^{\infty} \chi^{n}(\gamma)\left(l_{\gamma} \operatorname{csch} \frac{n l_{\gamma}}{2}\right) \exp \left(-\varepsilon\left(n l_{\gamma}\right)^{2}\right)
$$

is comparable in size to $2(\pi / \varepsilon)^{1 / 2} e^{1 / 16 \varepsilon}$.

Next take a sufficiently large finite partial sum of the last double sum so that the remainder is negligible in comparison with $2(\pi / \varepsilon)^{1 / 2} e^{1 / 16 \varepsilon}$. Then the remainder will continue to stay negligible in comparison with $2(\pi / \varepsilon)^{1 / 2} e^{1 / 16 \varepsilon}$ if we introduce a general $\chi$, since this only makes things smaller in absolute value.

Now the group $\Gamma$ has a presentation as a free group on $2 g$ ( $g=$ genus) generators, with one relation, namely that a certain product of commutators of the generators is the identity. This implies that we can assign any number of modulus 1 to each generator to get a character. If now we assign to each generator a number not equal to, but so close to 1 that for 
the resulting $\chi$,

$$
\sum_{\text {finite }} \chi^{n}(\gamma)\left(l_{\gamma} \operatorname{csch} \frac{n l_{\gamma}}{2}\right) \exp \left(-\varepsilon\left(n l_{\gamma}\right)^{2}\right)
$$

is comparable in size to $2(\pi / \varepsilon)^{1 / 2} e^{1 / 16 \varepsilon}$, we find, in view of what has been said, that some $r(\chi)$ must lie in $I_{1}$.

Having made this observation, let us now take $\chi$, which we will relabel $\chi_{1}$, to be the character that assigns $\exp \left(2 \pi i 2^{-N}\right)$ to each generator of $\Gamma$, where $N$ is a very large positive integer. Let $M$ be a large positive integer, but much smaller than $N$, and define the finite sequence of characters $\chi_{1}, \chi_{2}, \cdots, \chi_{M}$, by setting $\chi_{2}=\chi_{1}^{2}, \chi_{3}=\chi_{2}^{2}, \cdots, \chi_{M}=\chi_{M-1}^{2}$. Then no matter how large $M$ is, if $N$ is large enough, we can apply the previous argument to each $\chi_{1}, \chi_{2}, \cdots, \chi_{M}$ to get $r(\chi)$ 's in $I_{1}$ corresponding to eigenfunctions which transform by the characters $\chi_{1}, \chi_{2}, \cdots, \chi_{M}$, respectively.

At this point, we note that if $1 \leqq m \leqq M-1$, there exists $\gamma \in \Gamma$ such that $\chi_{m}(\gamma) \neq 1$, and $\chi_{n}(\gamma)=1$ for $n>m$.

Suppose now that $F_{1}(x), \cdots, F_{M}(x)$ are eigenfunctions for the problems corresponding to $\chi_{1}, \cdots, \chi_{M}$, with all having the corresponding $r(\chi)$ 's in $I_{1}$. Then $F_{1}(x), \cdots, F_{M}(x)$ are linearly independent, for if not, there exists an integer $k$ such that $1 \leqq k \leqq M-1$, a constant $c_{k} \neq 0$, and constants $c_{k+1}, \cdots, c_{M}$, such that

$$
c_{k} F_{k}(x)+c_{k+1} F_{k+1}(x)+\cdots+c_{M} F_{M}(x) \equiv 0 .
$$

Now suppose $\gamma \in \Gamma$ is chosen so that $\chi_{k}(\gamma) \neq 1$, but $\chi_{n}(\gamma)=1$ for $n>k$, and suppose $x_{0}$ is such that $F_{k}\left(x_{0}\right) \neq 0$. Then

$$
c_{k} F_{k}\left(\gamma x_{0}\right)+c_{k+1} F_{k+1}\left(\gamma x_{0}\right)+\cdots+c_{M} F_{M}\left(\gamma x_{0}\right)=0,
$$

or

$$
c_{k} \chi_{k}(\gamma) F_{k}\left(x_{0}\right)+c_{k+1} F_{k+1}\left(x_{0}\right)+\cdots+c_{M} F_{M}\left(x_{0}\right)=0 .
$$

Subtracting (3) from (2), with the latter specialized to $x=x_{0}$, we get $c_{k}\left(1-\chi_{k}(\gamma)\right) F_{k}\left(x_{0}\right)=0$, which is impossible.

Now let $\Gamma_{0}$ be the kernel in $\Gamma$ of the homomorphism $\chi_{1}$. Then $\Gamma_{0}$ is of finite index in $\Gamma$, since $\chi_{1}$ is a homomorphism into a finite group. Furthermore, for any $F_{i}(x)(i=1, \cdots, M), \gamma \in \Gamma_{0}$ implies that $F_{i}(\gamma x)=F_{i}(x)$. Thus the $F_{i}(x)$ 's are eigenfunctions of the Laplacian on the surface $\mathscr{S}_{0}$ corresponding to $\Gamma_{0}$, and since the $F_{i}(x)$ 's are linearly independent, we conclude that the number of $\lambda_{n}$ 's which lie in $\left[0, \frac{1}{4}\right)$ for the problem $\Delta F+$ $\lambda F=0$ on $\mathscr{S}_{0}$, counting multiplicities, is at least $M$. It is trivial to see that none of these eigenvalues is 0 , but even without that, the eigenvalue 0 occurs with multiplicity 1 , so we are done. 


\section{REFERENCES}

1. H. Huber, Zur analytischen Theorie hyperbolischer Raumformen und Bewegungsgruppen. II, Math. Ann. 142 (1960/61), 385-398. MR 23 \#A3845.

2. H. P. McKean, Selberg's trace formula as applied to a compact Riemann surface, Comm. Pure. Appl. Math. 25 (1972), 225-246.

3. A. Selberg, Harmonic analysis and discontinuous groups in weakly symmetric Riemannian spaces with applications to Dirichlet series, J. Indian Math. Soc. 20 (1956), 47-87. MR 19, 531.

4. B. Randol, On the analytic continuation of the Minakshisundaram-Pleijel zeta function for compact Riemann surfaces, Trans. Amer. Math. Soc. (to appear).

Department of Mathematics, City University of New York, Hunter College, NEW YoRK, NEW YORK 10021

City University of New York, Graduate School and University Center, 33 West 42 Street, New York, New York 10036 